\title{
PERIODIC BIFURCATION PROBLEMS \\ FOR FULLY NONLINEAR NEUTRAL FUNCTIONAL DIFFERENTIAL EQUATIONS \\ VIA AN INTEGRAL OPERATOR APPROACH: THE MULTIDIMENSIONAL DEGENERATION CASE
}

\author{
Jean-François Couchouron - Mikhail KamenskiI \\ Boris MikhaYlenKo - PAOLO Nistri
}

\begin{abstract}
We consider a $T$-periodically perturbed autonomous functional differential equation of neutral type. We assume the existence of a $T$ periodic limit cycle $x_{0}$ for the unperturbed autonomous system. We also assume that the linearized unperturbed equation around the limit cycle has the characteristic multiplier 1 of geometric multiplicity 1 and algebraic multiplicity greater than 1 . The paper deals with the existence of a branch of $T$-periodic solutions emanating from the limit cycle. The problem of finding such a branch is converted into the problem of finding a branch of zeros of a suitably defined bifurcation equation $P(x, \varepsilon)+\varepsilon Q(x, \varepsilon)=0$. The main task of the paper is to define a novel equivalent integral operator having the property that the $T$-periodic adjoint Floquet solutions of the unperturbed linearized operator correspond to those of the equation $P^{\prime}\left(x_{0}(\theta), 0\right)=0, \theta \in[0, T]$. Once this is done it is possible to express the condition for the existence of a branch of zeros for the bifurcation equation in terms of a multidimensional Malkin bifurcation function.
\end{abstract}

2010 Mathematics Subject Classification. Primary: 34K40, 34K27, 34K18, 34K13; Secondary: $47 \mathrm{G} 10,47 \mathrm{H} 08$.

Key words and phrases. Neutral functional differential equations, periodic perturbations, adjoint Floquet solutions, periodic bifurcation, multidimensional Malkin bifurcation function. 


\section{Introduction}

In [26] Malkin developed a perturbation theory to study the existence, uniqueness and stability of bifurcating periodic solutions from a limit cycle $x_{0}$ of an autonomous system when it is perturbed by a $T$-periodic nonlinear term of small amplitude. The system is of the form

$$
\dot{x}=\phi(x)+\varepsilon \psi(t, x, \varepsilon),
$$

where $\phi \in C^{2}\left(\mathbb{R}^{n}, \mathbb{R}^{n}\right), \psi \in C^{1}\left(\mathbb{R} \times \mathbb{R}^{n} \times[0,1], \mathbb{R}^{n}\right), \psi$ is $T$-periodic with respect to time and $\varepsilon \geq 0$ is a small parameter. The main tool for this study was the so-called Malkin bifurcation function

$$
f_{0}(\theta)=\int_{0}^{T}\left\langle z_{0}(\tau), \psi\left(\tau-\theta, x_{0}(\tau), 0\right)\right\rangle d \tau
$$

Here $\langle\cdot, \cdot\rangle$ denotes the scalar product in $\mathbb{R}^{n}$ and $z_{0}$ is a $T$-periodic solution of

$$
\dot{z}=\left(\phi^{\prime}\left(x_{0}(t)\right)\right)^{*} z,
$$

the adjoint system of the linearized unperturbed system

$$
\dot{y}=\phi^{\prime}\left(x_{0}(t)\right) y,
$$

which is assumed to have the characteristic multiplier 1 simple.

In [26] it is shown that if the bifurcation function $f_{0}$ has a simple zero $\theta_{0} \in[0, T]$, then there exists a branch of solutions $x_{\varepsilon}, \varepsilon \geq 0$ small, emanating from $x_{0}\left(\theta_{0}\right)$. In [27] Malkin extended the perturbation theory to periodically perturbed non autonomous $T$-periodic systems. The results obtained by Malkin in these papers have been also proved independently by Loud [22]. Since these pioneering papers [26], [27], [22] a relevant bibliography has been devoted to the refinement and development in various directions of the results contained in these papers, sometimes leading to different expressions and employ of the bifurcation function. From the huge bibliography on the subject we mention, in the sequel, some of the papers which are more closely related to the interest and methods employed in this paper.

Under the regularity assumptions on the functions $\phi$ and $\psi$ of (1.1) indicated above, in [16] the problem of finding a branch of $T$-periodic solutions originating from $x_{0}$ is reduced to the problem of finding a branch of zeros of a bifurcation equation of the form

$$
P(x)+\varepsilon Q(x, \varepsilon)=0,
$$

where $P: \mathbb{R}^{n} \rightarrow \mathbb{R}^{n}$ and $Q: \mathbb{R}^{n} \times[0,1] \rightarrow \mathbb{R}^{n}$ are given by $P(x)=\Pi_{0}(x)-x$ and $Q(x, \varepsilon)=\left(\Pi_{\varepsilon}(x)-\Pi_{0}(x)\right) / \varepsilon$ with $P^{\prime}\left(x_{0}\right)$ singular, here $\Pi_{\varepsilon}$ is the singular Poincaré map associated to (1.1). By means of the classical implicit function theorem, it is shown that, under the usual assumption of the existence of a simple zero of the Malkin bifurcation function associated to (1.1), the bifurcation 